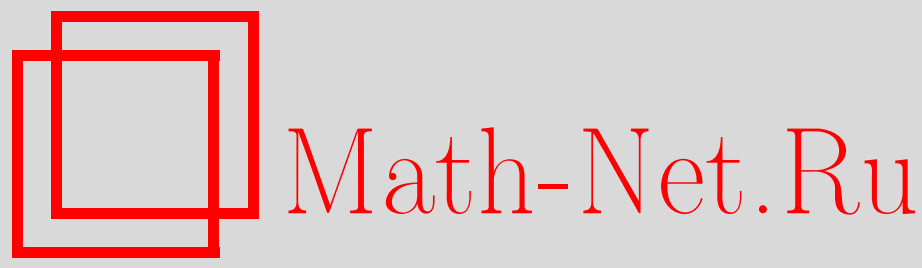

О. Н. Герман, Диофантовы экспоненты решеток, Совр. пробл. матем., 2016, выпуск 23, 35-42

DOI: https://doi.org/10.4213/spm60

Использование Общероссийского математического портала Math-Net.Ru подразумевает, что вы прочитали и согласны с пользовательским соглашением http://www . mathnet.ru/rus/agreement

Параметры загрузки:

IP: 54.172 .240 .79

26 апреля 2023 г., 13:55:54 


\title{
Диофантовы экспоненты решеток
}

\author{
О. Н. Герман \\ Математический институт им. В. А. Стеклова Российской академии наук, г. Москва \\ german.oleg@gmail.com
}

\section{1. Введение}

Существует множество задач, в которых естественным образом возникают разного рода диобантовы экспоненты. В самой общей постановке имеется $n$ линейно независимых линейных форм $\ell_{1}(\mathbf{z}), \ldots, \ell_{n}(\mathbf{z})$ от $d$ вещественных переменных, $n<d$. И вопрос заключается в том, насколько малым может быть набор $\left(\boldsymbol{\ell}_{1}(\mathbf{z}), \ldots, \boldsymbol{\ell}_{n}(\mathbf{z})\right)$, если $\mathbf{z}$ пробегает множество ненулевых точек с иелыми координатами. Есть два классических способа измерения «величины» этого набора: во-первых, можно рассматривать какою-нибудь норму, во-вторых - произведение модулей элементов. После чего исследуется вопрос, насколько быстро эта величина может стремиться к нулю с ростом «размера» $\mathbf{z}$.

Два примера для $\boldsymbol{n}<\boldsymbol{d}$. В качестве простейших примеров, иллюстрирующих два описанных выше подхода, можно привести задачу о совместных приближениях двух вещественных чисел и знаменитую гипотезу Литтлвуда (см. также [1], [2]). И там, и там речь идет о двух формах $\boldsymbol{\ell}_{1}(\mathbf{z}), \boldsymbol{\ell}_{2}(\mathbf{z})$ от трех переменных, коэффициенты которых записаны в строках матрицы

$$
\left(\begin{array}{lll}
\theta_{1} & 1 & 0 \\
\theta_{n} & 0 & 1
\end{array}\right)
$$

Пусть $|\cdot|$ обозначает sup-норму. Тогда супремум вещественных $\gamma$ таких, что неравенство

$$
\max _{i=1,2}\left|\boldsymbol{\ell}_{i}(\mathbf{z})\right| \leqslant|\mathbf{z}|^{-\gamma}
$$

имеет бесконечно много решений в $\mathbf{z} \in \mathbb{Z}^{3}$, называется диофантовой экспонентой пары $\left(\theta_{1}, \theta_{2}\right)$ и описывает, насколько хорошо можно одновременно приблизить $\theta_{1}$ и $\theta_{2}$ рациональными числами с одним и тем же знаменателем.

С другой стороны, знаменитая гипотеза Литтлвуда утверждает, что для любого $\varepsilon>0$ неравенство

$$
\prod_{i=1,2}\left|\ell_{i}(\mathbf{z})\right| \leqslant \varepsilon z_{1}^{-1}
$$

имеет бесконечно много решений в $\mathbf{z}=\left(z_{1}, z_{2}, z_{3}\right) \in \mathbb{Z}^{3}, z_{1} \neq 0$. Как и в случае совместных приближений, мультипликативная диофантова экспонента пары $\left(\theta_{1}, \theta_{2}\right)$ определяется как супремум вещественных $\gamma$ таких, что неравенство

$$
\prod_{i=1,2}\left|\ell_{i}(\mathbf{z})\right|^{1 / 2} \leqslant z_{1}^{-\gamma}
$$

имеет бесконечно много решений в $\mathbf{z}=\left(z_{1}, z_{2}, z_{3}\right) \in \mathbb{Z}^{3}, z_{1} \neq 0$. 
Случай $\boldsymbol{n}=\boldsymbol{d}$. В приведенных выше примерах было важно, что количество линейных форм строго меньше размерности объемлющего пространства. Благодаря этому условию область, в которой мы ищем целые точки, хотя бы не ограничена. Но если $n=d$, а формы линейно независимы, то «норменный» подход дает нам ограниченную область, параллелепипед, для которого можно определять что-нибудь типа последовательных минимумов, но никак не диофантовы экспоненты. Однако если рассматривать произведение координат, то все намного интереснее. В такой постановке мы приходим к понятию диофантовой экспоненты решетки.

\section{2. Экспоненты решеток}

Напомним (см. [3]), что гипотеза Литтлвуда тесно связана с так называемой гипотезой Оппенгейма для линейных форм, которая имеет дело с решеткой

$$
\Lambda=\left\{\left(\boldsymbol{\ell}_{1}(\mathbf{z}), \ldots, \boldsymbol{\ell}_{d}(\mathbf{z})\right) \mid \mathbf{z} \in \mathbb{Z}^{d}\right\},
$$

где $\boldsymbol{\ell}_{1}(\mathbf{z}), \ldots, \boldsymbol{\ell}_{d}(\mathbf{z})$ - линейно независимые линейные формы от $d$ переменных. Она утверждает, что при $d \geqslant 3$ величина

$$
N(\Lambda)=\inf _{\mathbf{z} \in \mathbb{Z}^{d} \backslash\{\mathbf{0}\}} \prod_{1 \leqslant i \leqslant d}\left|\ell_{i}(\mathbf{z})\right|,
$$

называемая норменным минимумом решетки $\Lambda$, положительна тогда и только тогда, когда $\Lambda$ эквивалентна по модулю действия группы диагональных матриц решетке полного модуля вполне вещественного алгебраического расширения $\mathbb{Q}$ степени $d$ (о таких алгебраических решетках см. [4]). Таким образом, если для каждого $\mathbf{x}=\left(x_{1}, \ldots, x_{d}\right) \in \mathbb{R}^{d}$ положить

$$
\Pi(\mathbf{x})=\prod_{1 \leqslant i \leqslant d}\left|x_{i}\right|^{1 / d}
$$

можно сказать, что гипотеза Оппенгейма предлагает критерий отделимости от нуля величины $\Pi(\mathbf{x})$ в ненулевых точках решетки $\Lambda$. Но если эта величина принимает сколь угодно близкие к нулю значения, то можно говорить о соответствующей диофантовой экспоненте. Как и прежде, через $|\cdot|$ будем обозначать sup-норму.

ОПРЕДЕЛЕНИЕ 1. Будем называть диофантовой экспонентой решетки $\Lambda$ супремум вещественных $\gamma$ таких, что неравенство

$$
\Pi(\mathbf{x}) \leqslant|\mathbf{x}|^{-\gamma}
$$

имеет бесконечно много решений в $\mathbf{x} \in \Lambda$. Обозначать эту величину будем $\omega(\Lambda)$.

Из теоремы Минковского о выпуклом теле следует, что для любой решетки $\Lambda$ справедливо тривиальное неравенство

$$
\omega(\Lambda) \geqslant 0 .
$$

В то же время $\omega(\Lambda)=0$ всякий раз, когда $N(\Lambda)>0$, например когда $\Lambda$ - решетка полного модуля вполне вещественного алгебраического расширения $\mathbb{Q}$, откуда, кстати, следует «достаточность» в утверждении гипотезы Оппенгейма.

Есть еще одно семейство решеток, для которых $\omega(\Lambda)=0$ в силу знаменитой теоремы о подпространствах, доказанной В. М. Шмидтом [5] в 1972 г. (см. также [6]).

Теорема 1 (теорема Шмидта о подпространствах, 1972). Eсли $\boldsymbol{\ell}_{1}(\mathbf{z}), \ldots, \boldsymbol{\ell}_{d}(\mathbf{z})$ - линейно независимые линейные формы от $d$ переменных с алгебраическими коэфбициентами, то для любого $\varepsilon>0$ существует лишь конечное число собственных подпространств $\mathbb{Q}^{d}$, содержащих все челочисленные решения неравенства

$$
\prod_{1 \leqslant i \leqslant d}\left|\ell_{i}(\mathbf{z})\right|<|\mathbf{z}|^{-\varepsilon} .
$$


СЛЕДСТвИЕ 1. Пусть $\ell_{1}(\mathbf{z}), \ldots, \ell_{d}(\mathbf{z})$ - линейно независимые линейные формы от $d$ переменных с алгебраическими коэфбициентами. Пусть также для любого $k$-набора $\left(i_{1}, \ldots, i_{k}\right)$, $1 \leqslant i_{1}<\cdots<i_{k} \leqslant d, 1 \leqslant k \leqslant d$, коэфбиииенты поливектора

$$
\ell_{i_{1}} \wedge \cdots \wedge \ell_{i_{k}}
$$

линейно независимы над $\mathbb{Q}$. Тогда для любого $\varepsilon>0$ существует лишь конечное число точек $\mathbf{z} \in \mathbb{Z}^{d} \operatorname{ma\kappa ux,~чmo~}$

$$
\prod_{1 \leqslant i \leqslant d}\left|\ell_{i}(\mathbf{z})\right|<|\mathbf{z}|^{-\varepsilon} .
$$

ДокАЗАТЕЛЬСтво. Из ограничения на коэффициенты следует, что в любом $k$-мерном рациональном подпространстве $\mathcal{L}$ пространства $\mathbb{R}^{d}$ любые $k$ из заданных линейных форм индуцируют $k$ линейно независимых линейных форм в $\mathcal{L}$.

Пусть теперь $\mathcal{L}$ - одно из рациональных подпространств, о которых идет речь в теореме о подпространствах. Можно считать, что $\operatorname{dim} \mathcal{L}=d-1$. Тогда ранг решетки $\mathcal{L} \cap \mathbb{Z}^{d}$ равен $d-1$. Выберем в этой решетке произвольный базис $\mathbf{b}_{1}, \ldots, \mathbf{b}_{d-1}$ и дополним его до базиса $\mathbb{Z}^{d}$ каким-нибудь вектором $\mathbf{b}_{d}$. Обозначим через $B$ матрицу, в столбцах которой записаны координаты векторов $\mathbf{b}_{1}, \ldots, \mathbf{b}_{d}$, и рассмотрим линейное преобразование с матрицей $B^{-1}$. При этом преобразовании $\mathcal{L}$ переходит в подпространство

$$
\left\{\mathbf{z}=\left(z_{1}, \ldots, z_{d}\right) \in \mathbb{R}^{d} \mid z_{d}=0\right\},
$$

естественно отождествляемое с $\mathbb{R}^{d-1}$, а решетка $\mathcal{L} \cap \mathbb{Z}^{d}-$ в решетку

$$
\left\{\mathbf{z}=\left(z_{1}, \ldots, z_{d}\right) \in \mathbb{Z}^{d} \mid z_{d}=0\right\},
$$

естественно отождествляемую с $\mathbb{Z}^{d-1}$.

Тогда исходные формы $\ell_{1}(\mathbf{z}), \ldots, \ell_{d}(\mathbf{z})$ индуцируют новые формы $\widetilde{\ell}_{1}(\widetilde{\mathbf{z}}), \ldots, \widetilde{\ell}_{d}(\widetilde{\mathbf{z}})$ от $d-1$-й переменной с алгебраическими коэффициентами, причем любые $d-1$ из этих форм линейно независимы. Существует константа $R$, зависящая лишь от коэффициентов этих форм такая, что множество

$$
\left\{\left.\widetilde{\mathbf{z}} \in \mathbb{R}^{d-1}\left|\prod_{1 \leqslant i \leqslant d}\right| \widetilde{\ell}_{i}(\widetilde{\mathbf{z}})|<| \widetilde{\mathbf{z}}\right|^{-\varepsilon},|\widetilde{\mathbf{z}}|>R\right\}
$$

содержится в объединении

$$
\bigcup_{1 \leqslant j \leqslant d}\left\{\left.\widetilde{\mathbf{z}} \in \mathbb{R}^{d-1}\left|\prod_{\substack{1 \leqslant i \leqslant d \\ i \neq j}}\right| \widetilde{\ell}_{i}(\widetilde{\mathbf{z}})|<| \widetilde{\mathbf{z}}\right|^{-\varepsilon}\right\} .
$$

Остается применить индукцию по размерности, ибо база ее - случай $d=2$ - очевидна.

СледСТВИЕ 2. Пусть линейные формы $\boldsymbol{\ell}_{1}(\mathbf{z}), \ldots, \boldsymbol{\ell}_{d}(\mathbf{z})-$ такие же, как в следствии 1 , и пусть $\Lambda$ - решетка, определяемая (1). Тогда

$$
\omega(\Lambda)=0 .
$$

ДокАзАтельство. Достаточно заметить, что

$$
|\mathbf{z}| \asymp \max _{1 \leqslant i \leqslant d}\left|\ell_{i}(\mathbf{z})\right|
$$

и применить следствие 1.

Возникает естественный вопрос: достигается ли каждое положительное значение $\omega(\Lambda)$. Однако соответствующие примеры еще предстоит построить. А пока мы хотели бы обратиться к принципу переноса. 


\section{3. Теорема переноса}

Пусть $\Lambda$ - произвольная решетка в $\mathbb{R}^{d}$ ранга $d$. Рассмотрим двойственную решетку

$$
\Lambda^{*}=\left\{\mathbf{y} \in \mathbb{R}^{d} \mid\langle\mathbf{y}, \mathbf{x}\rangle \in \mathbb{Z} \text { для любого } \mathbf{x} \in \Lambda\right\} \text {, }
$$

где $\langle\cdot, \cdot\rangle$ обозначает скалярное произведение. Оказывается, так же, как и во многих других задачах теории диофантовых приближений, можно доказывать теоремы переноса, т.е. утверждения, связывающие $\omega(\Lambda)$ и $\omega\left(\Lambda^{*}\right)$.

Конечно, при $d=2$ решетка $\Lambda^{*}$ совпадает с точностью до гомотетии с решеткой $\Lambda$, повернутой на $\pi / 2$, так что в двумерном случае справедливо очевидное равенство $\omega(\Lambda)=\omega\left(\Lambda^{*}\right)$.

Tеорема 2. Пусть $d \geqslant 3$. Тогда

$$
\omega(\Lambda) \geqslant \frac{\omega\left(\Lambda^{*}\right)}{(d-1)^{2}+d(d-2) \omega\left(\Lambda^{*}\right)} .
$$

Здесь имеется в виду, что при $\omega\left(\Lambda^{*}\right)=\infty$ справедливо

$$
\omega(\Lambda) \geqslant \frac{1}{d(d-2)} .
$$

Мы докажем теорему 2 при помощи понятия псевдоприсоединенного параллелепипеда (см. также [7]) и при помощи общей теоремы переноса, доказанной в [8]. Мы дадим соответствующее определение в самом простом случае, ибо этого случая нам вполне достаточно.

ОПРЕДЕЛЕНИЕ 2. Пусть $\eta_{1}, \ldots, \eta_{d}$ - положительные вещественные числа. Рассмотрим параллелепипед

$$
\mathcal{P}=\left\{\mathbf{x}=\left(x_{1}, \ldots, x_{d}\right) \in \mathbb{R}^{d}|| x_{i} \mid \leqslant \eta_{i}, i=1, \ldots, d\right\} .
$$

Тогда параллелепипед

$$
\mathcal{P}^{*}=\left\{\mathbf{x}=\left(x_{1}, \ldots, x_{d}\right) \in \mathbb{R}^{d}|| x_{i} \mid \leqslant \frac{1}{\eta_{i}} \prod_{1 \leqslant j \leqslant d} \eta_{j}, i=1, \ldots, d\right\}
$$

называется псевдоприсоединенным к П.

Принцип переноса, открытый Хинчиным [9] в одном частном случае, привел в итоге к следующему довольно общему наблюдению (см. [8]).

Теорема 3 (Г., Евдокимов, 2015). Пусть $c=d^{1 / 2(d-2)} u \operatorname{det} \Lambda=1$. Пусть $\mathcal{P} u \mathcal{P}^{*}-\kappa a \kappa$ в определении 2. Тогда

$$
\mathcal{P}^{*} \cap \Lambda^{*} \neq\{\mathbf{0}\} \quad \Longrightarrow \quad c \mathcal{P} \cap \Lambda \neq\{\mathbf{0}\} .
$$

Выведем теорему 2 из теоремы 3.

ДОКАЗАТЕЛЬСТВо ТЕОРЕмЫ 2. Поскольку $\omega(\Lambda)$ инвариантна относительно гомотетий, можно считать, что $\operatorname{det} \Lambda=1$. Мы рассмотрим два случая.

Случай I. На координатных плоскостях нет ненулевых точек решетки $\Lambda^{*}$.

Зафиксируем произвольное положительное $\varepsilon$. Тогда найдется бесконечно много ненулевых точек $\mathbf{u}=\left(u_{1}, \ldots, u_{d}\right) \in \Lambda^{*}$ таких, что

$$
\Pi(\mathbf{u})=|\mathbf{u}|^{-\gamma}, \quad \gamma=\gamma(\mathbf{u}) \geqslant \begin{cases}\omega\left(\Lambda^{*}\right)-\varepsilon & \text { при } \omega\left(\Lambda^{*}\right)<\infty, \\ \frac{1}{\varepsilon} & \text { при } \omega\left(\Lambda^{*}\right)=\infty .\end{cases}
$$


Рассмотрим любую из этих точек и положим

$$
\mathcal{P}_{\mathbf{u}}=\left\{\mathbf{x}=\left(x_{1}, \ldots, x_{d}\right) \in \mathbb{R}^{d}|| x_{i}|\leqslant| u_{i} \mid, i=1, \ldots, d\right\} .
$$

Поскольку все $u_{i}$ отличны от нуля, множество $\mathcal{P}_{\mathbf{u}}$ является невырожденным параллелепипедом. Более того, $\mathcal{P}_{\mathbf{u}}=\mathcal{P}^{*}$ для

$$
\mathcal{P}=\left\{\mathbf{x}=\left(x_{1}, \ldots, x_{d}\right) \in \mathbb{R}^{d}|| x_{i} \mid \leqslant \eta_{i}, i=1, \ldots, d\right\},
$$

где

$$
\eta_{i}=\left|u_{i}\right|^{-1} \prod_{1 \leqslant j \leqslant d}\left|u_{j}\right|^{1 /(d-1)} .
$$

Стало быть, по теореме 3 параллелепипед $c \mathcal{P}$ содержит ненулевую точку $\mathbf{v}=\left(v_{1}, \ldots, v_{d}\right)$ решетки $\Lambda$. Для этой точки имеем

$$
\begin{aligned}
|\mathbf{v}| \leqslant c \max _{1 \leqslant i \leqslant d}\left|\eta_{i}\right| \leqslant c \cdot \frac{\prod_{1 \leqslant i \leqslant d}\left|\eta_{i}\right|}{\min _{1 \leqslant i \leqslant d}\left|\eta_{i}\right|^{d-1}}=c \cdot \frac{\max _{1 \leqslant i \leqslant d}\left|u_{i}\right|^{d-1}}{\prod_{1 \leqslant i \leqslant d}\left|u_{i}\right|^{d-2 /(d-1)}} \\
=c \cdot \frac{|\mathbf{u}|^{d-1}}{\Pi(\mathbf{u})^{d(d-2) /(d-1)}}=c|\mathbf{u}|^{d-1+d(d-2) /(d-1) \gamma}
\end{aligned}
$$

и

$$
\Pi(\mathbf{v}) \leqslant c \cdot \prod_{1 \leqslant i \leqslant d}\left|\eta_{i}\right|^{1 / d}=c \cdot \prod_{1 \leqslant i \leqslant d}\left|u_{i}\right|^{1 / d(d-1)}=c \Pi(\mathbf{u})^{1 /(d-1)}=c|\mathbf{u}|^{-\gamma /(d-1)} .
$$

Таким образом,

$$
\Pi(\mathbf{v}) \leqslant c_{1}|\mathbf{v}|^{-\frac{\gamma}{(d-1)^{2}+d(d-2) \gamma}}, \quad c_{1}=c_{1}(d, \gamma) .
$$

Отметим, что $|\mathbf{u}|$ может принимать сколь угодно большие значения. Стало быть, $\min _{1 \leqslant i \leqslant d}\left|\eta_{i}\right|$ может принимать сколь угодно малые значения, ибо

$$
\min _{1 \leqslant i \leqslant d}\left|\eta_{i}\right|=\max _{1 \leqslant i \leqslant d}\left|u_{i}\right|^{-1} \prod_{1 \leqslant j \leqslant d}\left|u_{j}\right|^{1 /(d-1)}=|\mathbf{u}|^{-1} \Pi(\mathbf{u})^{d /(d-1)}=|\mathbf{u}|^{-1-d \gamma /(d-1)} .
$$

Это наблюдение в случае отсутствия в координатных плоскостях ненулевых точек решетки $\Lambda$ дает нам бесконечно много точек $\Lambda$, удовлетворяющих (5), откуда немедленно следует (4). Если же в какой-нибудь координатной плоскости все же есть ненулевая точка решетки $\Lambda$, то, очевидно, $\omega(\Lambda)=\infty$, и тогда неравенство (4) тривиально.

Случай II. На координатных плоскостях имеются ненулевые точки решетки $\Lambda^{*}$.

В этом случае $\omega\left(\Lambda^{*}\right)=\infty$, и нам нужно показать, что

$$
\omega(\Lambda) \geqslant \frac{1}{d(d-2)} .
$$

Можно считать, что в решетке $\Lambda^{*}$ имеется ненулевая точка $\mathbf{u}=\left(u_{1}, \ldots, u_{d}\right)$ такая, что $u_{d}=0$. Тогда $(d-1)$-мерное подпространство, ортогональное $\mathbf{u}$, содержит последнюю координатную ось, а также некоторую подрешетку $\Gamma \subset \Lambda$ ранга $d-1$. Применяя теорему Минковского о выпуклом теле, получаем, что существует бесконечно много точек $\mathbf{v}=\left(v_{1}, \ldots, v_{d}\right) \in \Gamma$, $v_{d} \rightarrow \infty$ таких, что

$$
\max _{1 \leqslant i \leqslant d-1}\left|v_{i}\right| \leqslant c_{2}\left|v_{d}\right|^{-1 /(d-2)}, \quad c_{2}=c_{2}(\mathbf{u})
$$

Для каждой такой точки $\mathbf{v}$ справедливо

$$
\Pi(\mathbf{v}) \leqslant\left(c_{2}^{d-1}\left|v_{d}\right|^{1-(d-1) /(d-2)}\right)^{1 / d}=c_{2}^{(d-1) / d}|\mathbf{v}|^{-1 / d(d-2)},
$$

откуда немедленно следует (6). 


\section{4. На пути к спектру}

Как и в других диофантовых задачах (см. [10]-[12]), естественно возникает вопрос описания соответствующего спектра - подмножества $(\mathbb{R} \cup\{\infty\})^{2}$, заметаемого парами $\left(\omega(\Lambda), \omega\left(\Lambda^{*}\right)\right)$, если $\Lambda$ пробегает все пространство решеток ранга $d$, содержащихся в $\mathbb{R}^{d}$.

Как мы уже заметили, при $d=2$ справедливо равенство $\omega(\Lambda)=\omega\left(\Lambda^{*}\right)$. Кроме того, в этом простейшем случае все можно описать в терминах цепных дробей (см. [13], [14]), так что в случае $d=2$ легко убедиться, что все неотрицательные значения $\omega(\Lambda)$ достигаются.

При $d \geqslant 3$ имеют место неравенства

$$
\omega(\Lambda) \geqslant 0, \quad \omega\left(\Lambda^{*}\right) \geqslant 0, \quad \omega(\Lambda) \geqslant \frac{\omega\left(\Lambda^{*}\right)}{(d-1)^{2}+d(d-2) \omega\left(\Lambda^{*}\right)},
$$

и интересен вопрос, все ли точки задаваемого ими множества принадлежат спектру пары $\left(\omega(\Lambda), \omega\left(\Lambda^{*}\right)\right)$.

Пока что известно довольно мало. Мы знаем примеры решеток $\Lambda$, для которых $\omega(\Lambda)=$ $\omega\left(\Lambda^{*}\right)=0$. Это решетки с положительным норменным минимумом (2) и решетки, удовлетворяющие условиям следствий 1 и 2. Действительно, с одной стороны, известно (см. [15]-[17]), что

$$
N(\Lambda)>0 \Longleftrightarrow N\left(\Lambda^{*}\right)>0 .
$$

С другой стороны, если $\ell_{1}(\mathbf{z}), \ldots, \ell_{d}(\mathbf{z})$ линейно независимы и если $\ell_{1}^{*}(\mathbf{z}), \ldots, \ell_{d}^{*}(\mathbf{z})-$ двойственный набор форм, то коэффициенты поливектора $\boldsymbol{\ell}_{i_{1}}(\mathbf{z}) \wedge \cdots \wedge \boldsymbol{\ell}_{i_{k}}(\mathbf{z})$ совпадают с точностью до знака с коэффициентами поливектора $\ell_{i_{k+1}}^{*}(\mathbf{z}) \wedge \cdots \wedge \boldsymbol{\ell}_{i_{d}}^{*}(\mathbf{z})$, где $\left(i_{1}, \ldots, i_{d}\right)$ - перестановка из $(1, \ldots, d)$. Стало быть, эти наборы коэффициентов одновременно являются линейно независимыми над $\mathbb{Q}$, откуда следует, что если $\Lambda$ удовлетворяет условию следствия 1 , то ему удовлетворяет и $\Lambda^{*}$.

Оказывается, при помощи теоремы о подпространствах можно привести примеры решеток $\Lambda$, для которых

$$
\omega(\Lambda)=\frac{1}{d(d-2)}, \quad \omega\left(\Lambda^{*}\right)=\infty,
$$

доказав таким образом точность теоремы 2 в одном граничном случае.

ТЕОРема 4. Пусть $\ell_{1}(\mathbf{z}), \ldots, \ell_{d}(\mathbf{z})$ - линейно независимые линейные формы от $d$ nеременных с алгебраическими коэфбициентами. Пусть первый коэффициент поливектора

$$
\ell_{1} \wedge \cdots \wedge \ell_{d-1}
$$

равен нулю, а все остальные линейно независимы над $\mathbb{Q}$. Пусть также для любого $k$-набора $\left(i_{1}, \ldots, i_{k}\right)$, отличного от $(1, \ldots, d-1), 1 \leqslant i_{1}<\cdots<i_{k} \leqslant d, 1 \leqslant k \leqslant d$, коэфбициентв поливектора

$$
\ell_{i_{1}} \wedge \cdots \wedge \ell_{i_{k}}
$$

линейно независимь над $\mathbb{Q}$. Наконеи, пусть $\Lambda$ - решетка, определяемая (1). Тогда

$$
\omega(\Lambda)=\frac{1}{d(d-2)}, \quad \omega\left(\Lambda^{*}\right)=\infty
$$

ДокАЗАТЕЛЬСтво. Поскольку первый коэффициент $\boldsymbol{\ell}_{1} \wedge \cdots \wedge \boldsymbol{\ell}_{d-1}$ равен нулю, в плоскости

$$
\mathcal{L}_{1}=\left\{\mathbf{z}=\left(z_{1}, \ldots, z_{d}\right) \in \mathbb{R}^{d} \mid z_{d}=0\right\}
$$

есть ненулевая точка решетки $\Lambda^{*}$. Следовательно, $\omega\left(\Lambda^{*}\right)=\infty$, и в силу теоремы 2 достаточно показать, что

$$
\omega(\Lambda) \leqslant \frac{1}{d(d-2)}
$$


Из условия следует, что если $\mathcal{L}$ - произвольное рациональное подпространство $\mathbb{R}^{d}$, отличное от $\mathcal{L}_{1}, \operatorname{dim} \mathcal{L}=k$, то любые $k$ из исходных форм индуцируют $k$ линейно независимых линейных форм в $\mathcal{L}$. Стало быть, повторяя рассуждение из доказательства следствия 1, можно показать, что для любого $\varepsilon>0$ и любого $\mathbf{z} \in \mathbb{Z}^{d} \backslash \mathcal{L}_{1}$ выполняется

$$
\prod_{1 \leqslant i \leqslant d}\left|\boldsymbol{\ell}_{i}(\mathbf{z})\right| \geqslant c_{3}|\mathbf{z}|^{-\varepsilon}, \quad c_{3}=c_{3}\left(\varepsilon, \boldsymbol{\ell}_{1}, \ldots, \boldsymbol{\ell}_{d}\right) .
$$

Что же касается $\mathcal{L}_{1}$, по условию любые $d-1$ форм

$$
\boldsymbol{\ell}_{i_{1}}(\mathbf{z}), \ldots, \boldsymbol{\ell}_{i_{d-2}}(\mathbf{z}), \boldsymbol{\ell}_{d}(\mathbf{z}), \quad 1 \leqslant i_{1}<\cdots<i_{d-2} \leqslant d-1,
$$

индуцируют линейно независимые линейные формы $\widetilde{\ell}_{1}(\widetilde{\mathbf{z}}), \ldots, \widetilde{\ell}_{d-1}(\widetilde{\mathbf{z}})$ в $\mathcal{L}_{1}$, удовлетворяющие условию следствия 1. Следовательно, для любого $\varepsilon>0$, любого $j \in\{1, \ldots, d-1\}$ и любого ненулевого $\mathbf{z} \in \mathcal{L}_{1}$ справедливо

$$
\prod_{\substack{1 \leqslant i \leqslant d \\ i \neq j}}\left|\ell_{i}(\mathbf{z})\right| \geqslant c_{4}|\mathbf{z}|^{-\varepsilon}, \quad c_{4}=c_{4}\left(\varepsilon, \ell_{1}, \ldots, \ell_{d}\right) .
$$

Отсюда

$$
\left|\boldsymbol{\ell}_{d}(\mathbf{z})\right|\left(\prod_{1 \leqslant i \leqslant d}\left|\boldsymbol{\ell}_{i}(\mathbf{z})\right|\right)^{d-2}=\prod_{1 \leqslant j \leqslant d-1}\left(\prod_{\substack{1 \leqslant i \leqslant d \\ i \neq j}}\left|\boldsymbol{\ell}_{i}(\mathbf{z})\right|\right) \geqslant c_{4}^{d-1}|\mathbf{z}|^{-(d-1) \varepsilon}
$$

Таким образом, учитывая (3), получаем, что для каждого $\varepsilon>0$ и каждого ненулевого $\mathbf{z} \in \mathcal{L}_{1}$ справедливо

$$
\prod_{1 \leqslant i \leqslant d}\left|\ell_{i}(\mathbf{z})\right| \geqslant c_{5}|\mathbf{z}|^{-1 /(d-2)-\varepsilon}, \quad c_{5}=c_{5}\left(\varepsilon, \ell_{1}, \ldots, \ell_{d}\right) .
$$

Учитывая еще раз (3), получаем из (9) и (10), что для любого $\varepsilon>0$ и любого $\mathbf{x} \in \Lambda$ справедливо

$$
\Pi(\mathbf{x}) \geqslant c_{6}|\mathbf{x}|^{-1 / d(d-2)-\varepsilon}, \quad c_{6}=c_{6}(\varepsilon, \Lambda),
$$

откуда немедленно следует (8).

Несложно видеть, что аналогичное рассуждение можно применить для построения решеток таких, что $\omega\left(\Lambda^{*}\right)=\infty$, а $\omega(\Lambda)$ равно любому из значений из серии

$$
\frac{k(d-k-l)}{d l}, \quad k \in\{1, \ldots, d-2\}, \quad l \in\{1, \ldots, d-k-1\} .
$$

Чтобы построить соответствующую решетку, нужно рассмотреть линейные формы $\boldsymbol{\ell}_{1}(\mathbf{z}), \ldots$, $\boldsymbol{\ell}_{d}(\mathbf{z})$ с алгебраическими коэффициентами такими, что $k$-мерное подпространство

$$
\ell_{1}(\mathbf{z})=\cdots=\ell_{d-k}(\mathbf{z})=0
$$

содержится в рациональном подпространстве размерности $k+l \leqslant d-1$, но не содержится ни в каком рациональном подпространстве меньшей размерности. Тогда теорема Минковского о выпуклом теле даст неравенство

$$
\omega(\Lambda) \geqslant \frac{k(d-k-l)}{d l},
$$

а теорема о подпространствах - обратное.

Однако числа (11) - единственные нетривиальные значения $\omega(\Lambda)$, которые дает этот метод. Существование же решеток с конечной ненулевой экспонентой, отличной от (11), пока не доказано. 


\section{Список литературы}

[1] J. W. S. Cassels, An Introduction to the Geometry of Numbers, Springer-Verlag, Berlin, 1997.

[2] P. M. Gruber, C. G. Lekkerkerker, Geometry of Numbers, North-Holland Math. Library, 37, North-Holland Publ., Amsterdam, 1987.

[3] J. W.S. Cassels, H.P. F. Swinnerton-Dyer, "On the product of three homogeneous linear forms and indefinite ternary quadratic forms", Philos. Trans. Roy. Soc. London. Ser. A, 248 (1955), 73-96.

[4] A. I. Borevich, I. R. Shafarevich, Number Theory, Pure Appl. Math., 20, Academic Press, New York, 1966.

[5] W. M. Schmidt, "Norm form equations", Ann. of Math. (2), 96 (1972), 526-551.

[6] E. Bombieri, W. Gubler, Heights in Diophantine Geometry, New Math. Monogr., 4, Cambridge Univ. Press, Cambridge, 2006.

[7] W. M. Schmidt, Diophantine Approximation, Lecture Notes in Math., 785, Springer-Verlag, Berlin, 1980.

[8] О.Н. Герман, К. Г. Евдокимов, "Усиление теоремы переноса Малера", Изв. РАН. Сер. матем., 79:1 (2015), 63-76.

[9] A. Ya. Khintchine, "Über eine Klasse linearer Diophantischer Approximationen", Rend. Sirc. Mat. Palermo, 50:2 (1926), 170-195.

[10] M. Laurent, "Exponents of Diophantine approximation in dimension two", Canad. J. Math., 61:1 (2009), 165-189.

[11] A. Marnat, About Jarnik's-Type Relation in Higher Dimension, arXiv: 1510.06334.

[12] A. Marnat, "There is no analogue to Jarnik's relation for twisted diophantine approximation", Monats. Math., 181 (2016), 675-688, arXiv: 1409.6665.

[13] J.W.S. Cassels, An Introduction to Diophantine Approximation, Cambridge Tracts in Math. and Math. Phys., 45, Cambridge Univ. Press, Cambridge, 1957.

[14] A. Ya. Khintchine, Continued Fractions, Dover Publ., Mineola, NY, 1997.

[15] О.Н. Герман, "Паруса и норменные минимумы решеток", Матем. сб., 196:3 (2005), 31-60.

[16] O. N. German, "Klein polyhedra and lattices with positive norm minima", J. Theor. Nombres Bordeaux, 19:1 (2007), 175-190.

[17] Б. Ф. Скубенко, "Минимумы разложимых форм степени $n$ от $n$ переменных при $n \geqslant 3 "$, Moдулярные функции и квадратичные формы. 1, Зап. научн. сем. ЛОМИ, 183, Изд-во «Наука», Ленинград. отд., Л., 1990, 142-154. 